\title{
Incursion Recognition Mechanism Based On Secure Network System
}

\author{
A. Mohamed Divan Masood', S.K. Muthusundar ${ }^{2}$ \\ ${ }^{1}$ Information Technology, AMET University, Chennai, India \\ ${ }^{2}$ Department of computer science, Sri Muthukumaran Institute of Technology, India
}

\begin{tabular}{|c|c|}
\hline Article Info & ABSTRACT \\
\hline Article history: & Internet based computing dissimilar services such as server storage and \\
\hline Received Nov 21, 2017 & $\begin{array}{l}\text { applications are shared on the internet. This makes cloud computing one of } \\
\text { the most promising and rapidly growing technologies. As it relies on sharing }\end{array}$ \\
\hline Revised Jan 29, 2018 & computer resources, it is prone to various security risks. Individual such \\
\hline Accepted Feb 17, 2018 & $\begin{array}{l}\text { security issue is Distributed Denial of Services attack on cloud. A DDos } \\
\text { assault can begin from anyplace in the system and normally overpowers the }\end{array}$ \\
\hline Keywords: & $\begin{array}{l}\text { casualty server by sending countless. This paper deals with the prevention of } \\
\text { DDos attacks and how honey pot approach can be used in cloud computing } \\
\text { to counter DDos attacks. }\end{array}$ \\
\hline
\end{tabular}

Distributed Denial of Services

\section{Corresponding Author:}

A. Mohamed Divan Masood, Information Technology, AMET University, Chennai, India.

\section{INTRODUCTION}

Denial of service (DoS) attacks has been one of the major network security problems over the last decades. DoS attacks can usually be mounted on hardware devices such as routers and firewalls to send spoofing messages to the target network [1]. Thus, methods for defeating such DoS attacks are highly related to the vulnerabilities in the hardware devices [2]. In this paper, we investigate the potential attacks specific to the hardware infrastructure of the network and also categorize the countermeasures against DoS attacks that can be implemented on hardware devices [3]. Moreover, we analyze the advantages of the emerging silicon physical unsolvable functions and discuss the potential of integrating them into authentication methods in order to defend against DoS attacks [4].

Recently used as an attacking platform to form larger scale of flooding DDoS attacks, and attack flows become more distributed and even more harmful making it increasingly hard to be detected effectively DDoS attacks are identified, if a server or network is already down or exhausted for a while [5].

It is difficult to distinguish the legitimate packets on normal traffic and packets sent by computers is Media Access Delay and Throughput Analysis of Voice Codec with Silence Suppression on Wireless Ad Hoc Network [6]. Hence, there is a lag in DDoS attack detection. As large number of packets is transmitted, more time is required to analyze each incoming packet is Optimal Scheduling Based on Instance Niche for Channel Assignment in Ad-Hoc Network Optimal Scheduling Based On Instance Niche for Channel Assignment in Ad-Hoc Network [7]. 


\section{PROPOSED SYSTEM}

An efficient hop-by-hop honey pot mechanism is proposed into mitigate the spoofing distributed DoS attacks. Here back propagation is performed to trace back the root of attacks. In addition, roaming honey pots scheme provides accurate attack signatures Total energy Efficiency of cellular large scale antenna system multiple access mobile networks [8]. On receiving attack packets, the roaming honey pot triggers the activation of a tree of honey pot sessions rooted at the honey pot under the attack toward attack sources. To reduce the delay progressive back propagation is used to handle low-rate attacks such as on-off attacks with short bursts.

However there is no security system to protect the honey pots from unknown attacks false negatives false positives and as a result happening. If an attacker breaks into honey pot it will break the honey pot connections and make it a bouncer.

We have proposed ADTRVH where the virtual roaming honey pot is used along with the multi-level secure architecture to collect the information regarding various intruders at different levels in the network.

\section{ARCHITECTURE DIAGRAM}

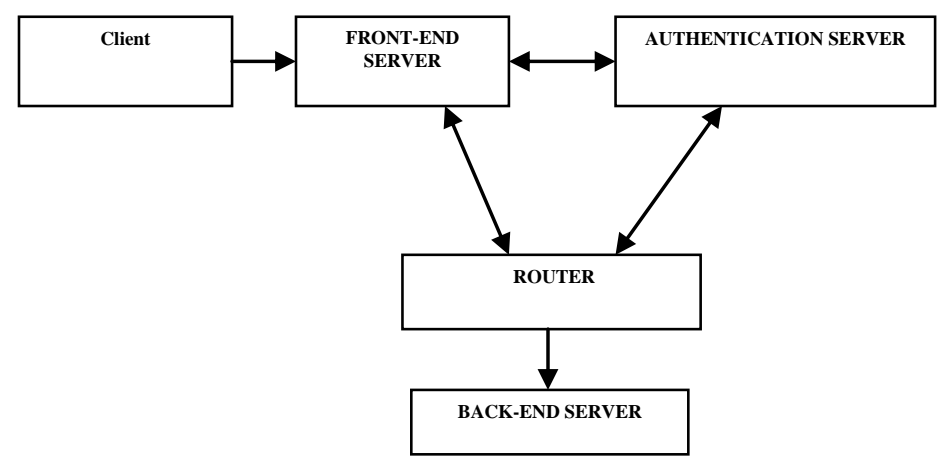

\section{RESULT AND DISCUSSION}

The present invention further contemplates that in some instances it may be desirous to disable certain tasks regardless of memory or processor utilization. Such an instance could occur, for example, if a user wished to disable all attack signatures made irrelevant by the network information discovered on the network .Although the present invention has been described in detail, it should be understood that various changes, substitutions and alterations can be made thereto without departing from the spirit and scope of the invention as defined by the appended claims.

\section{CONCLUSION}

In this work, we investigated the idea of nectar pots inside and out and perceived how it may be valuable to the field of system security. The idea of nectar pots is an essential expansion to the security field. Nectar pots offer a hostile way to deal with interruption recognition and shirking. Above all they fill in as a learning apparatus for framework overseers and furthermore included considering issues concerning interruption location frameworks the difficulties that these frameworks confronted. The Web has turned out to be fundamental both at the various leveled and individual level in this way it will be the circumstance with security structure. The usage of nectar pots and related developments is on the rising. As care and excitement for nectar pots fabricates so will its usage in a relationship as a security gadget.

\section{REFERENCES}

[1] Yasser Alosefer, Omer Rena. Nectar item: an electronic low participation client honey pot. Third IEEE International Conference on Software Testing, Verification, and Validation Workshops (ICSTW). 2010.

[2] Xiao a Sun, et al. Collecting Internet Malware Based on Client-side Honey pot. ${ }^{\text {th }}$ IEEE International Conference for Young Computer Scientists (ICVCS 2008). 2008.

[3] C H Nick Jap, et al. The use of honey pot approach in software-based application protection for shareware programs, IEEE International Conference on Computing \& Informatics. (ICOCI '06) 2006.

[4] Jian Boaet al. Look into on system security of resistance in light of Nectar pot. IEEE International Conference on Computer Application and System Modeling. (ICCASM). 2010. 
[5] R C Joshi. Nectar pot Based Guiding to Direct DDoS Ambushes on Servers at ISP Level. IEEE International Symposiums on Information Processing (ISIP). 2008.

[6] Shah R D, Singh S K. Media Access Delay and Throughput Analysis of Voice Codec with Silence Suppression on Wireless Ad Hoc Network. Procedia Computer Science. 2016; 79; 940-947.

[7] Arivazhagan D, Helen D. Optimal Scheduling Based On Instance Niche for Channel Assignment in Ad-Hoc Network.

[8] H. Yang, et al, "Total energy Efficiency of cellular large scale antenna system multiple access mobile networks," In Proc. IEEE Online Green Comm, 2013, pp. 27-32 